\title{
1D DYNAMICS OF A SECOND-GRADE VISCOUS FLUID IN A CONSTRICTED TUBE
}

\author{
FERNANDO CARAPAU \\ Dept. Matemática and CIMA/UE, Universidade de Évora \\ Rua Romão Ramalho 59, 7001 Évora, Portugal \\ E-mail: flc@uevora.pt \\ ADÉLIA SEQUEIRA \\ Dept. Matemática and CEMAT/IST \\ Instituto Superior Técnico, Universidade Técnica de Lisboa \\ Av. Rovisco Pais, 1049 Lisboa, Portugal \\ E-mail: adelia.sequeira@math.ist.utl.pt
}

\begin{abstract}
Using a one-dimensional hierarchical model based on the Cosserat theory approach to fluid dynamics we can reduce the full 3D system of equations for the axisymmetric unsteady motion of a non-Newtonian incompressible second-grade viscous fluid to a system of equations depending on time and on a single spatial variable. From this new system we obtain the steady relationship between average pressure gradient and volume flow rate over a finite section of a straight constricted tube, and the corresponding equation for the wall shear stress.
\end{abstract}

1. Introduction. In this work we introduce a $1 \mathrm{D}$ hierarchical model for non-Newtonian Rivlin-Ericksen fluids of second-grade in an axisymmetric straight, rigid and impermeable constricted tube, based on the nine-directors theory approach developed by Caulk and Naghdi [7].

The Cosserat theory includes an additional structure of directors (deformable vectors) assigned to each point on a space curve (Cosserat curve), where a three-dimensional system of equations is replaced by a one-dimensional system depending on time and on a single spatial variable, obtained by integrating the equation of conservation of linear

2000 Mathematics Subject Classification: Primary 76A05; Secondary 76M25.

Key words and phrases: Cosserat theory, volume flow rate, average pressure gradient, wall shear stress, constricted tube.

Research of the authors are supported by CIMA/UE and CEMAT/IST, under FCT's funding program.

The paper is in final form and no version of it will be published elsewhere. 
momentum over the cross section of the tube. The relevance of using a theory of directed curves is not in regarding it as an approximation to three-dimensional equations, but rather in their use as an independent theory to predict some of the main properties of the corresponding three-dimensional problem. Advantages of the director theory include, in particular: the theory incorporates all components of the linear momentum; it is a hierarchical theory, allowing to increase the accuracy of the model; there is no need to include extra assumptions to close the system; the material invariance principle is satisfied at each order and the wall shear stress enters directly in the formulation as a dependent variable.

The use of directors in continuum mechanics goes back to Duhen [10] who regards a body as a collection of points together with associated directions. For additional background information and historical development of this theory related with studies of rods, plates and shells, see e.g. [9], [17], [18] and with fluid dynamics, see e.g. [7], [14], [15], [16]. Recently, this theory has also been applied in haemodynamics to model blood flow in the vascular system, see [2], [6], [20]. The same theory has been developed for different models in the case of a uniform rectilinear tube, see [3], [4], [5].

Taking into account [7], the fluid velocity field $\boldsymbol{v}=v_{i} \boldsymbol{e}_{i}$ can be approximated by:

$$
\boldsymbol{v}=\boldsymbol{v}^{*}+\sum_{N=1}^{k} x_{\alpha_{1}} \ldots x_{\alpha_{N}} \boldsymbol{W}_{\alpha_{1} \ldots \alpha_{N}}
$$

with

$$
\boldsymbol{v}^{*}=v_{i}^{*}(z, t) \boldsymbol{e}_{i}, \quad \boldsymbol{W}_{\alpha_{1} \ldots \alpha_{N}}=W_{\alpha_{1} \ldots \alpha_{N}}^{i}(z, t) \boldsymbol{e}_{i},
$$

(Latin subscripts take the values 1,2,3, Greek subscripts 1,2, and the usual summation convention is employed over a repeated index). Here, $\boldsymbol{v}^{*}$ represents the velocity along the axis of symmetry $z$ at time $t, x_{\alpha_{1}} \ldots x_{\alpha_{N}}$ are the polynomial weighting functions with order $k$ (this number identifies the order of hierarchical theory and is related to the number of directors), the vectors $\boldsymbol{W}_{\alpha_{1} \ldots \alpha_{N}}$ are the director velocities which are symmetric with respect to their indices and $\boldsymbol{e}_{i}$ are the associated unit basis vectors.

The aim of this paper is to apply the nine-director theory $(k=3$ in equation (1)) to a second-grade fluid model to obtain the steady relationship between average pressure gradient and volume flow rate over a finite section of a straight constricted tube. The corresponding equation for the wall shear stress is given and the theory is illustrated with numerical results for different values of Reynolds and Weissenberg numbers.

2. Equations of motion. Let us consider a homogeneous fluid moving within a straight and impermeable constricted tube $\Omega$ with circular cross-section contained in $\mathbb{R}^{3}$ (see Figure 1), where the surface function

$$
\phi(z)=\beta+\beta z^{2}, \quad \beta>0
$$

is related with the cross-section of the tube by the following relationship

$$
\phi^{2}(z)=x_{1}^{2}+x_{2}^{2} .
$$

Its boundary $\partial \Omega$ is composed by the proximal cross-section $\Gamma_{1}$, the distal cross-section 


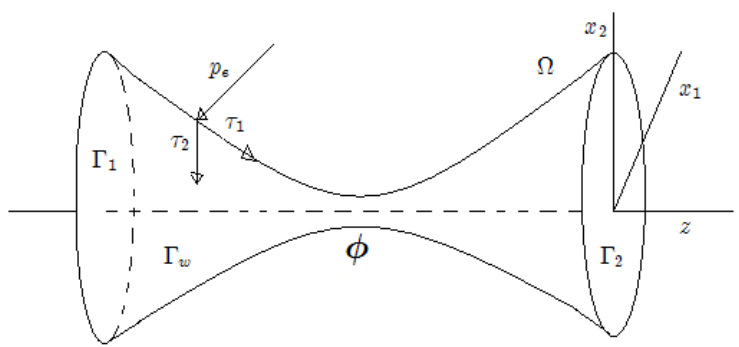

Fig. 1. Domain $\Omega$ with surface function given by (3), where the tangential components of the surface traction vector are $\tau_{1}, \tau_{2}$ and $p_{e}$. The rectangular cartesian coordinates are denoted by $x_{i}(i=1,2,3)$ and for convenience we set $x_{3}=z$.

$\Gamma_{2}$ and the lateral wall of the tube, denoted by $\Gamma_{w}$. The three-dimensional equations governing the axisymmetric motion of an incompressible second-grade viscous fluid, without body forces, is given by

$$
\left\{\begin{array}{l}
\rho\left(\frac{\partial \boldsymbol{v}}{\partial t}+\boldsymbol{v} \cdot \nabla \boldsymbol{v}\right)=\nabla \cdot \boldsymbol{T}, \quad \text { in } \Omega \times(0, T), \\
\nabla \cdot \boldsymbol{v}=0, \\
\boldsymbol{T}=-p \boldsymbol{I}+\boldsymbol{\sigma}, \boldsymbol{t}=\boldsymbol{T} \cdot \boldsymbol{n},
\end{array}\right.
$$

with the initial condition

$$
\boldsymbol{v}(x, 0)=\boldsymbol{v}_{0}(x) \text { in } \Omega,
$$

and the homogeneous Dirichlet boundary condition

$$
\boldsymbol{v}(x, t)=0 \text { on } \Gamma_{w} \times(0, T),
$$

where $p$ is the pressure, $-p \boldsymbol{I}$ is the spherical part of the stress due to the constraint of incompressibility and $\rho$ is the constant fluid density. Equation $(5)_{1}$ represents the balance of linear momentum and $(5)_{2}$ is the incompressibility condition. In equation $(5)_{3}, \boldsymbol{T}$ is the Cauchy stress tensor, $\boldsymbol{t}$ denotes the Cauchy stress tensor on the surface whose outward unit normal is $\boldsymbol{n}$, and $\boldsymbol{\sigma}$ is the extra stress tensor, given by

$$
\boldsymbol{\sigma}=\mu \boldsymbol{A}_{1}+\alpha_{1} \boldsymbol{A}_{2}+\alpha_{2} \boldsymbol{A}_{1}^{2},
$$

where $\mu$ is the constant fluid viscosity, $\alpha_{1}$ and $\alpha_{2}$ are material coefficients usually called the normal stress moduli and the kinematic first two Rivlin-Ericksen tensors $\boldsymbol{A}_{1}$ and $\boldsymbol{A}_{2}$ are given by (see Rivlin and Ericksen [19])

$$
\boldsymbol{A}_{1}=\nabla \boldsymbol{v}+(\nabla \boldsymbol{v})^{T}
$$

and

$$
\boldsymbol{A}_{2}=\frac{\partial}{\partial t}\left(\boldsymbol{A}_{1}\right)+\boldsymbol{v} \cdot \nabla \boldsymbol{A}_{1}+\boldsymbol{A}_{1} \nabla \boldsymbol{v}+(\nabla \boldsymbol{v})^{T} \boldsymbol{A}_{1} .
$$

If the fluid modelled by the extra stress tensor (8) is to be compatible with thermodynamics in the sense that all motions of the fluid meet the Clausius-Duhem inequality and the assumption that the specific Helmholtz free energy of the fluid is a minimum in 
equilibrium, then (see e.g. Giesekus [13])

$$
\mu \geqslant 0, \quad \alpha_{1} \geqslant 0, \quad \alpha_{1}+\alpha_{2}=0 .
$$

The fluids characterized by those restrictions are known as second-grade fluids as opposed to the general second-order fluids. The condition $(11)_{3}$ simplifies substantially the mathematical model and the corresponding analysis. Here we shall consider this particular case. If $\alpha_{1}=\alpha_{2}=0$ in equation (8), the classical Navier-Stokes system are recovered. This system of equations (5) - (10) has been studied by several authors (see e.g. [1], [8], [11], [12]) under different perspectives and in different domains.

The study of 1D models involves averaged quantities such as volume flow rate and average pressure. Therefore, let us consider $S(z, t)$ as a generic axial section of the tube at time $t$ defined by the spatial variable $z$, bounded by the cross-section of the constricted tube defined in (4) and let $A(z, t)$ be the area of this section $S(z, t)$. Then, the volume flow rate $Q$ is defined by

$$
Q(z, t)=\int_{S(z, t)} v_{3}\left(x_{1}, x_{2}, z, t\right) d a
$$

and the average pressure $\bar{p}$, by

$$
\bar{p}(z, t)=\frac{1}{A(z, t)} \int_{S(z, t)} p\left(x_{1}, x_{2}, z, t\right) d a .
$$

It follows from Caulk and Naghdi [7] that the approximation of the three-dimensional velocity field $\boldsymbol{v}$, using nine directors, i.e. $k=3$ in equation (1), is given by

$$
\begin{aligned}
\boldsymbol{v} & =\left[x_{1}\left(1-\frac{x_{1}^{2}+x_{2}^{2}}{\phi^{2}}\right) \frac{2 \phi_{z} Q}{\pi \phi^{3}}\right] \boldsymbol{e}_{1}+\left[x_{2}\left(1-\frac{x_{1}^{2}+x_{2}^{2}}{\phi^{2}}\right) \frac{2 \phi_{z} Q}{\pi \phi^{3}}\right] \boldsymbol{e}_{2} \\
& +\left[\frac{2 Q}{\pi \phi^{2}}\left(1-\frac{x_{1}^{2}+x_{2}^{2}}{\phi^{2}}\right)\right] \boldsymbol{e}_{3}
\end{aligned}
$$

where the subscript variable denotes partial differentiation and the volume flow rate $Q(t)$ is

$$
Q(t)=\frac{\pi}{2} \phi^{2}(z) v_{3}^{*}(z, t) .
$$

Also, from Caulk and Naghdi [7] the stress vector on the lateral surface $\Gamma_{w}$, using nine directors, is given by

$$
\begin{aligned}
\boldsymbol{t}_{w} & =\left[\frac{1}{\phi\left(1+\phi_{z}^{2}\right)^{1 / 2}}\left(\tau_{1} x_{1} \phi_{z}-p_{e} x_{1}-\tau_{2} x_{2}\left(1+\phi_{z}^{2}\right)^{1 / 2}\right)\right] \boldsymbol{e}_{1} \\
& +\left[\frac{1}{\phi\left(1+\phi_{z}^{2}\right)^{1 / 2}}\left(\tau_{1} x_{2} \phi_{z}-p_{e} x_{2}+\tau_{2} x_{1}\left(1+\phi_{z}^{2}\right)^{1 / 2}\right)\right] \boldsymbol{e}_{2} \\
& +\left[\frac{1}{\left(1+\phi_{z}^{2}\right)^{1 / 2}}\left(\tau_{1}+p_{e} \phi_{z}\right)\right] \boldsymbol{e}_{3}
\end{aligned}
$$

where $\tau_{1}, \tau_{2}$ and $p_{e}$ are the tangential components of the surface traction vector. 
Instead of satisfying the momentum equation $(5)_{1}$ pointwise in the fluid, we impose the following integral conditions

$$
\begin{gathered}
\int_{S(z, t)}\left[\nabla \cdot \boldsymbol{T}-\rho\left(\frac{\partial \boldsymbol{v}}{\partial t}+\boldsymbol{v} \cdot \nabla \boldsymbol{v}\right)\right] d a=0 \\
\int_{S(z, t)}\left[\nabla \cdot \boldsymbol{T}-\rho\left(\frac{\partial \boldsymbol{v}}{\partial t}+\boldsymbol{v} \cdot \nabla \boldsymbol{v}\right)\right] x_{\alpha_{1}} \ldots x_{\alpha_{N}} d a=0,
\end{gathered}
$$

where $N=1,2,3$.

Using the divergence theorem and integration by parts, equations (17) - (18) for nine directors, can be reduced to the four vector equations:

$$
\frac{\partial \boldsymbol{n}}{\partial z}+\boldsymbol{f}=\boldsymbol{a}, \quad \frac{\partial \boldsymbol{m}^{\alpha_{1} \ldots \alpha_{N}}}{\partial z}+\boldsymbol{l}^{\alpha_{1} \ldots \alpha_{N}}=\boldsymbol{k}^{\alpha_{1} \ldots \alpha_{N}}+\boldsymbol{b}^{\alpha_{1} \ldots \alpha_{N}},
$$

where $\boldsymbol{n}, \boldsymbol{k}^{\alpha_{1} \ldots \alpha_{N}}, \boldsymbol{m}^{\alpha_{1} \ldots \alpha_{N}}$ are resultant forces defined by

$$
\begin{gathered}
\boldsymbol{n}=\int_{S} \boldsymbol{t}_{3} d a, \quad \boldsymbol{k}^{\alpha}=\int_{S} \boldsymbol{t}_{\alpha} d a, \boldsymbol{k}^{\alpha \beta}=\int_{S}\left(\boldsymbol{t}_{\alpha} x_{\beta}+\boldsymbol{t}_{\beta} x_{\alpha}\right) d a, \\
\boldsymbol{k}^{\alpha \beta \gamma}=\int_{S}\left(\boldsymbol{t}_{\alpha} x_{\beta} x_{\gamma}+\boldsymbol{t}_{\beta} x_{\alpha} x_{\gamma}+\boldsymbol{t}_{\gamma} x_{\alpha} x_{\beta}\right) d a \\
\boldsymbol{m}^{\alpha_{1} \ldots \alpha_{N}}=\int_{S} \boldsymbol{t}_{3} x_{\alpha_{1}} \ldots x_{\alpha_{N}} d a .
\end{gathered}
$$

The quantities $\boldsymbol{a}$ and $\boldsymbol{b}^{\alpha_{1} \ldots \alpha_{N}}$ are inertia terms written as follows

$$
\begin{gathered}
\boldsymbol{a}=\int_{S} \rho\left(\frac{\partial \boldsymbol{v}}{\partial t}+\boldsymbol{v} \cdot \nabla \boldsymbol{v}\right) d a \\
\boldsymbol{b}^{\alpha_{1} \ldots \alpha_{N}}=\int_{S} \rho\left(\frac{\partial \boldsymbol{v}}{\partial t}+\boldsymbol{v} \cdot \nabla \boldsymbol{v}\right) x_{\alpha_{1}} \ldots x_{\alpha_{N}} d a
\end{gathered}
$$

and $f, l^{\alpha_{1} \ldots \alpha_{N}}$, which arise due to surface traction on the lateral boundary, are given by

$$
\boldsymbol{f}=\int_{\partial S}\left(1+\phi_{z}^{2}\right)^{1 / 2} \boldsymbol{t}_{w} d s, \quad \boldsymbol{l}^{\alpha_{1} \ldots \alpha_{N}}=\int_{\partial S}\left(1+\phi_{z}^{2}\right)^{1 / 2} \boldsymbol{t}_{w} x_{\alpha_{1}} \ldots x_{\alpha_{N}} d s
$$

The equation relating the mean pressure gradient (wall shear stress, respectively) with the volume flow rate will be obtained using these quantities.

3. Numerical results. Let us consider a straight and rigid walled constricted tube with surface function given by (3). Replacing the steady results (20) - (25) obtained for the nine-director model into equations (19), and using the dimensionless variables

$$
\hat{z}=\frac{z}{\phi_{0}}, \quad \hat{\phi}=\frac{\phi}{\phi_{0}}, \quad \hat{Q}=\frac{2 \rho}{\pi \phi_{0} \mu} Q, \quad \hat{\bar{p}}=\frac{\phi_{0}^{2} \rho}{\mu^{2}} \bar{p},
$$

where $\phi_{0}$ is a characteristic radius of the constricted tube, we get the following steady relationship

$$
\hat{\bar{p}}_{\hat{z}}=-4 \frac{\hat{B}_{1}}{\hat{\phi}^{4}} \hat{Q}-\left(\frac{\hat{B}_{2}}{\hat{\phi}^{5}}+\mathcal{W}_{e}\left(\frac{\hat{B}_{3}}{\hat{\phi}^{7}}+\varepsilon \frac{\hat{B}_{4}}{\hat{\phi}^{7}}\right)\right) \hat{Q}^{2},
$$

where $\mathcal{W}_{e}=\alpha_{1} /\left(\rho \phi_{0}^{2}\right), \varepsilon=\alpha_{2} / \alpha_{1}$ are viscoelastic parameters and the nondimensional flow rate $\hat{Q}$ is identical to the classical Reynolds number, see e.g. [20]. The nondimen- 
sional number $\mathcal{W}_{e}$ is also called the Weissenberg number, see [12]. When we neglect the viscoelastic effect $\mathcal{W}_{e}$, the fluid becomes purely viscous. Flow separation occurs when the axial component $\tau_{1}$ of the stress vector on the lateral surface is in the direction of the flow, i.e. $\tau_{1}>0$. Then, the expression for the dimensionless form of the wall shear stress $\hat{\tau}_{1}$ is given by

$$
\hat{\tau}_{1}=-2 \hat{B}_{5} \hat{Q}-\frac{1}{6}\left(\hat{B}_{6}+\mathcal{W}_{e}\left(\hat{B}_{7}+\varepsilon \hat{B}_{8}\right)\right) \hat{Q}^{2} .
$$

Using a polynomial approximation of order $\mathcal{O}\left(\hat{z}^{5}\right)$ of equations $(27)-(28)$, the nondimensional functions $\hat{B}_{1}, \hat{B}_{2}, \hat{B}_{3}, \hat{B}_{4}, \hat{B}_{5}, \hat{B}_{6}$ and $\hat{B}_{7}, \hat{B}_{8}$ are given by

$$
\begin{gathered}
\hat{B}_{1}=1+\frac{2}{3} \hat{\beta}^{2}+\frac{1}{8} \hat{\beta}^{4}+\left(2 \hat{\beta}^{2}+\frac{9}{4} \hat{\beta}^{4}\right) \hat{z}^{2}+\frac{25}{8} \hat{\beta}^{4} \hat{z}^{4} \\
\hat{B}_{2}=\left(-2 \hat{\beta}-\frac{3}{10} \hat{\beta}^{3}\right) \hat{z}-\frac{3}{10} \hat{\beta}^{3} \hat{z}^{3} \\
\hat{B}_{3}=\left(8 \hat{\beta}-\frac{352}{3} \hat{\beta}^{3}+\frac{8}{3} \hat{\beta}^{5}\right) \hat{z}+\left(\frac{944}{3} \hat{\beta}^{3}-\frac{376}{3} \hat{\beta}^{5}\right) \hat{z}^{3} \\
\hat{B}_{4}=\left(20 \hat{\beta}-\frac{176}{3} \hat{\beta}^{3}\right) \hat{z}+\left(\frac{544}{3} \hat{\beta}^{3}-48 \hat{\beta}^{5}\right) \hat{z}^{3} \\
\hat{B}_{5}=a c 1 \hat{\beta}^{3}+\frac{2}{3 \hat{\beta}}+\frac{1}{8 \hat{\beta}}+\left(-\frac{4}{\hat{\beta}}+\frac{5}{24} \hat{\beta}-\frac{3}{\hat{\beta}^{3}}-\frac{1}{2} \hat{\beta}^{3}\right) \hat{z}^{2} \\
+\left(\frac{89}{8} \hat{\beta}+\frac{10}{\hat{\beta}}-\frac{5}{6} \hat{\beta}^{3}+\frac{6}{\hat{\beta}^{3}}+2 \hat{\beta}^{5}\right) \hat{z}^{4} \\
\hat{B}_{6}=\left(-\frac{2}{\hat{\beta}^{3}}-\frac{19}{10 \hat{\beta}}\right) \hat{z}+\left(\frac{177}{10 \hat{\beta}}+\frac{8}{\hat{\beta}^{3}}+\frac{38}{5} \hat{\beta}\right) \hat{z}^{3} \\
\hat{B}_{7}=\left(-\frac{144}{\hat{\beta}^{3}}-\frac{30}{\hat{\beta}}\right) \hat{z}+\left(\frac{1312}{\hat{\beta}^{3}}+\frac{576}{\hat{\beta}^{3}}+120 \hat{\beta}\right) \hat{z}^{3}
\end{gathered}
$$

and

$$
\hat{B}_{8}=\left(-\frac{24}{\hat{\beta}^{3}}-\frac{18}{\hat{\beta}}\right) \hat{z}+\left(\frac{232}{\hat{\beta}^{3}}+\frac{240}{\hat{\beta}}+72 \hat{\beta}\right) \hat{z}^{3} .
$$

Now, let us consider the interval $\left[\hat{z}_{1}, \hat{z}\right]$, where $\hat{z}_{1}$ is fixed. Integrating the equation (27) over the section $\left[\hat{z}_{1}, \hat{z}\right]$, we obtain the steady nondimensional equation for average pressure gradient with nine directors given by

$$
\hat{p p}(\hat{z})=4 \hat{A}_{1}(\hat{z}) \hat{Q}+\left(\hat{A}_{2}(\hat{z})+\mathcal{W}_{e}\left(\hat{A}_{3}(\hat{z})+\varepsilon \hat{A}_{4}(\hat{z})\right)\right) \hat{Q}^{2}
$$

where

$$
\hat{A}_{1}(\hat{z})=-\int_{\hat{z}_{1}}^{\hat{z}}\left(\frac{\hat{B}_{1}}{\hat{\phi}^{4}}\right) d \hat{z}, \quad \hat{A}_{2}(\hat{z})=-\int_{\hat{z}_{1}}^{\hat{z}}\left(\frac{\hat{B}_{2}}{\hat{\phi}^{5}}\right) d \hat{z}
$$

and

$$
\hat{A}_{3}(\hat{z})=-\int_{\hat{z}_{1}}^{\hat{z}}\left(\frac{\hat{B}_{3}}{\hat{\phi}^{7}}\right) d \hat{z}, \quad \hat{A}_{4}(\hat{z})=-\int_{\hat{z}_{1}}^{\hat{z}}\left(\frac{\hat{B}_{4}}{\hat{\phi}^{7}}\right) d \hat{z} .
$$

Since in this model $\alpha_{1}+\alpha_{2}=0$, then $\varepsilon=-1$ in the equations (28) and (29). Next, 
considering $\beta=0.1$ in equation (3), we compare the average pressure gradient (29) (wall shear stress (28), respectively) for different values of Reynolds and Weissenberg numbers.
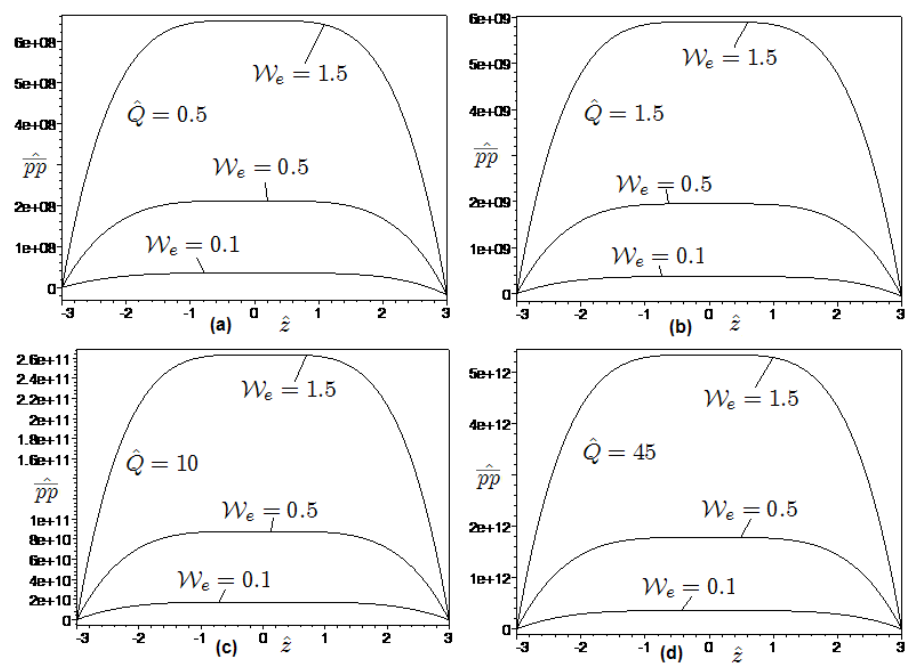

Fig. 2. Nondimensional average pressure gradient (29) obtained by the nine-directors theory for different values of the Weissenberg $\mathcal{W}_{e}=(0.1,0.5,1.5)$ and Reynolds numbers $(a) \hat{Q}=0.5 ;(b)$ $\hat{Q}=1.5 ;(c) \hat{Q}=10$ and $(d) \hat{Q}=45$.

Shown in Figure 2 and Figure 3 is the nondimensional average pressure gradient (29) for different values of the Reynolds and Weissenberg numbers. In these figures, we observe the same behavior of the average pressure gradient when we increase the values of the Reynolds number with fixed Weissenberg number, and vice-versa. The wall shear stress undergoes remarkable oscillations for small increasing values of the Reynolds number with fixed Weissenberg number, and vice-versa, as observed in Figure 4 and Figure 5.
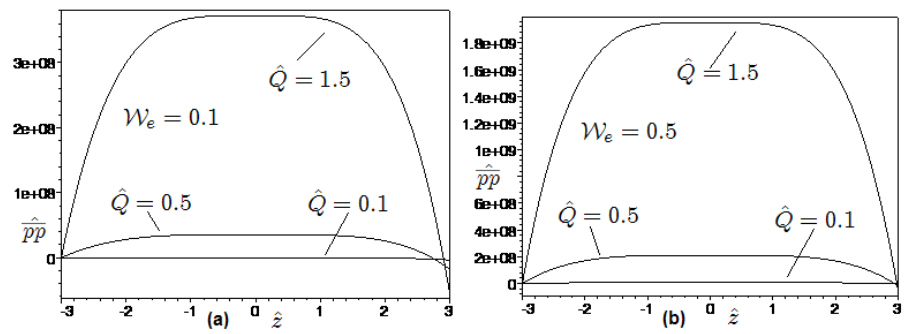

Fig. 3. Nondimensional average pressure gradient (29) obtained by the nine-directors theory for different values of the Reynolds $\hat{Q}=(0.1,0.5,1.5)$ and Weissenberg numbers $(a) \mathcal{W}_{e}=0.1$ and (b) $\mathcal{W}_{e}=0.5$.

Several numerical tests have been performed for other values of $\beta>0$, Reynolds $(\hat{Q} \gg 100)$ and Weissenberg numbers, showing similar qualitative results of the average pressure gradient and wall shear stress solutions. 

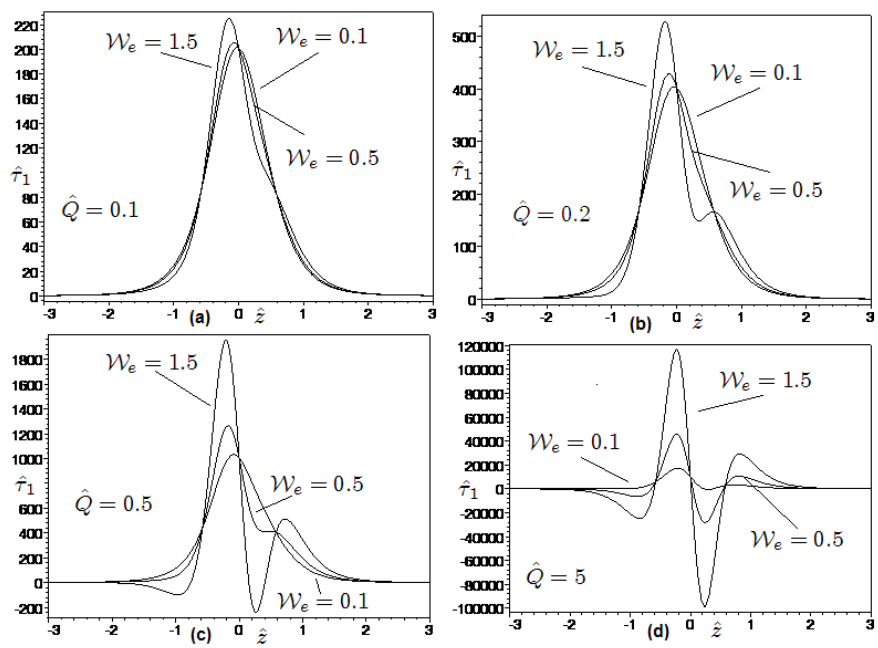

Fig. 4. Nondimensional wall shear stress (28) obtained by the nine-directors theory for different values of the Weissenberg $\mathcal{W}_{e}=(0.1,0.5,1.5)$ and Reynolds numbers $(a) \hat{Q}=0.1 ;(b) \hat{Q}=0.2$; (c) $\hat{Q}=0.5$ and $(d) \hat{Q}=5$.
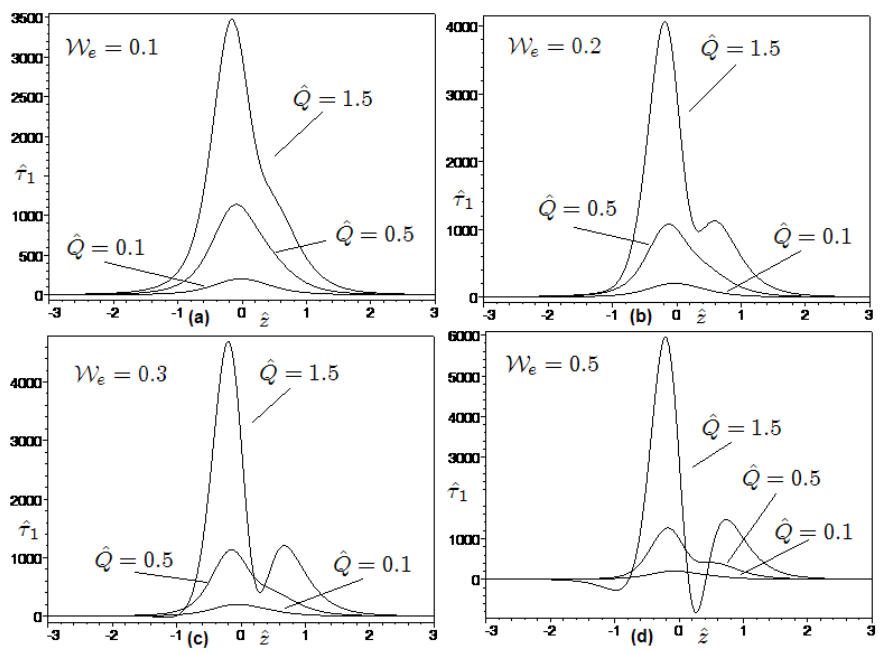

Fig. 5. Nondimensional wall shear stress (28) obtained by the nine-directors theory for different values of the Reynolds $\hat{Q}=(0.1,0.5,1.5)$ and Weissenberg numbers $(a) \mathcal{W}_{e}=0.1 ;(b) \mathcal{W}_{e}=0.2$; (c) $\mathcal{W}_{e}=0.3$ and $(d) \mathcal{W}_{e}=0.5$

4. Conclusions. The $1 \mathrm{D}$ Cosserat model provides an independent and alternative theory to predict some of the main properties of associated three-dimensional problems. In this work, we apply the theory to second-grade fluids in a constricted tube and show the behavior of the average pressure gradient and wall shear stress solutions for different values of Reynolds and Weissenberg numbers. One of the possible extensions of this work is the application of this 1D irector approach to study fluid-structure interaction problems. 


\section{References}

[1] P. H. Boulanger, H. Hayes and K. R. Rajagopal, Some unsteady exact solutions in the navier-Stokes and the second-grade fluid theories, SAACM 1 (1991), 185-203.

[2] F. Carapau, Development of 1D fluid models using the Cosserat theory. Numerical simulations and applications to haemodynamics, PhD Thesis, IST, Lisbon, Portugal, 2005.

[3] F. Carapau and A. Sequeira, Unsteady flow of a generalized Oldroyd-B fluid using a director theory approach, WSEAS Transactions on Fluid Mechanics 1 (2006), 167-174.

[4] F. Carapau and A. Sequeira, Axisymmetric motion of a second order viscous fluid in a circular straight tube under pressure gradients varying exponentially with time, in: Advances in Fluid Mechanics VI, WIT Trans. Eng. Sci. 52, WIT Press, Southampton, 2006, 409-419.

[5] F. Carapau, A. Sequeira and J. Janela, 1D simulations of second-grade fluids with sheardependent viscosity, WSEAS Transactions on Mathematics 6 (2007), 151-158.

[6] F. Carapau and A. Sequeira, 1D Models for blood flow in small vessels using the Cosserat theory, WSEAS Transactions on Mathematics 5 (2006), 54-62.

[7] D. A. Caulk and P. M. Naghdi, Axisymmetric motion of a viscous fluid inside a slender surface of revolution, Journal of Applied Mechanics 54 (1987), 190-196.

[8] V. Coscia and G. P. Galdi, Existence, uniqueness and stability of regular steady motions of a second-grade fluid, Int. J. Non-Linear Mechanics 29 (1994), 493-506.

[9] E. Cosserat and F. Cosserat, Sur la théorie des corps minces, Compt. Rend. 146 (1908), 169-172.

[10] P. Duhem, Le potentiel thermodynamique et la pression hydrostatique, Ann. École Norm. 10 (1893), 187-230.

[11] G. P. Galdi and A. Sequeira, Further existence results for classical solutions of the equations of a second-grade fluid, Arch. Rational Mech. Anal. 128 (1994), 297-312.

[12] G. P. Galdi, A. Vaidya, M. Pokorný, D. Joseph and J. Feng, Orientation of symmetric bodies falling in a second-order liquid at nonzero Reynolds number, Mathematical Models and Methods in Applied Sciences 12 (2002), 1653-1690.

[13] H. Giesekus, Die Simultane Translations and Rotations Bewegung einer Kugel in einer Elastovisken Flussigkeit, Rheol. Acta 3 (1963), 59-71.

[14] A. E. Green and P. M. Naghdi, A direct theory of viscous fluid flow in pipes I. Basic general developments, Phil. Trans. R. Soc. Lond. A 342 (1993), 525-542.

[15] A. E. Green and P. M. Naghdi, A direct theory of viscous fluid flow in pipes II. Flow of incompressible viscous fluid in curved pipes, Phil. Trans. R. Soc. Lond. A 342 (1993), 543-572.

[16] A. E. Green and P. M. Naghdi, A direct theory of viscous fluid flow in channels, Arch. Ration. Mech. Analysis 86 (1984), 39-63.

[17] A. E. Green, N. Laws and P. M. Naghdi, Rods, plates and shells, Proc. Camb. Phil. Soc. 64 (1968), 895-913.

[18] A. E. Green, P. M. Naghdi and M. L. Wenner, On the theory of rods II. Developments by direct approach, Proc. R. Soc. Lond. A 337 (1974), 485-507.

[19] R. S. Rivlin and J. L. Ericksen, Stress-deformation relations for isotropic materials, J. Rational Mech. Anal. 4 (1955), 323-425.

[20] A. M. Robertson and A. Sequeira, A director theory approach for modeling blood flow in the arterial system: An alternative to classical 1D models, Mathematical Models \& Methods in Applied Sciences 15 (2005), 871-906. 
